\title{
Age-dependent increased sag current in human pyramidal neurons dampens baseline cortical activity
}

\author{
Alexandre Guet-McCreight ${ }^{1}$, Homeira Moradi Chameh ${ }^{2}$, Sara Mahallati ${ }^{2,6}$, Margaret Wishart ${ }^{1}$, \\ Shreejoy J. Tripathy ${ }^{1,3,4}$, Taufik A. Valiante ${ }^{2,4,5,6,7,8,9}$, Etay Hay ${ }^{1,3,10}$ \\ ${ }^{1}$ Krembil Centre for Neuroinformatics, Centre for Addiction and Mental Health ${ }^{2}$ Krembil Brain Institute, University Health Network ${ }^{3}$ Department \\ of Psychiatry, University of Toronto ${ }^{4}$ Institute of Medical Sciences, University of Toronto ${ }^{5}$ Department of Electrical and Computer Engineering, \\ University of Toronto ${ }^{6}$ Institute of Biomaterials and Biomedical Engineering, University of Toronto ${ }^{7}$ Department of Surgery, University of \\ Toronto ${ }^{8}$ Center for Advancing Neurotechnological Innovation to Application ${ }^{9}$ Max Planck-University of Toronto Center for Neural Science and \\ Technology ${ }^{10}$ Department of Physiology, University of Toronto
}

\begin{abstract}
Aging involves various neurobiological changes, although their effect on brain function in humans remains poorly understood. The growing availability of human neuronal and circuit data provides opportunities for uncovering age-dependent changes of brain networks and for constraining models to predict consequences on brain activity. Here we found increased sag current in human layer 5 pyramidal neurons from older subjects, and captured this effect in biophysical models of younger and older pyramidal neurons. We used these models to simulate detailed layer 5 microcircuits and found lower baseline firing in older pyramidal neuron microcircuits, with minimal effect on response. We then validated the predicted reduced baseline firing using extracellular multi-electrode recordings from human brain slices of different ages. Our results thus report changes in human pyramidal neuron input integration properties and provide fundamental insights on the neuronal mechanisms of altered cortical excitability and resting state activity in human aging.
\end{abstract}

Corresponding authors: Dr. Etay Hay and Dr. Alexandre Guet-McCreight Krembil Centre for Neuroinformatics, Centre for Addiction and Mental Health 250 College St, Toronto, Ontario, M5T 1R8

Email:

etay.hay@,camh.ca

alexandre.guet-mccreight@camh.ca 


\section{Introduction}

Aging of the human brain is a variable and multifaceted process in terms of cognitive, cellular and anatomical changes (Peters, 2006). Among the different age-associated declines in cognitive performance, older people often exhibit slower cortical processing (Bucur \& Madden, 2010) and reduced discrimination acuity (Legge et al., 2008, 2019). Whereas the underlying cellular and circuit mechanisms that cause these deficits remain unknown, studies in monkeys indicate that a decline in working memory with age is associated with reduced baseline and response spike rates in prefrontal cortex, which may involve increased hyperpolarization-activated cyclic nucleotidegated (HCN) current, or h-current (Ramos et al., 2006; Wang et al., 2007, 2011). The increasing availability of human neuronal transcriptomic and electrophysiological datasets (Gouwens et al., 2018; Hodge et al., 2019; Chameh et al., 2021; Goriounova et al. 2018) offers an opportunity to look for neuronal changes across age demographics and, through computational modeling, determine their impacts on signal processing and behavior.

In cortical pyramidal neurons, h-current modulates signal integration, particularly in the apical dendrites due to increased density with distance from soma (Kole et al., 2006). Increased hcurrent, which is the primary contributor to generating and shaping sag voltage, results in a more depolarized resting membrane potential (Williams \& Stuart, 2000; Beaulieu-Laroche et al., 2018) and decreased input resistance (Song \& Moyer, 2017), which consequentially dampens excitatory post-synaptic potential (PSP) summation (Williams \& Stuart, 2000; Beaulieu-Laroche et al., 2018). Moreover, increased sag voltage amplitude and depolarized resting membrane potentials have been reported in pyramidal neurons of deeper layers in human cortex (Chameh et al., 2021). Previous studies suggest age-associated changes in h-current which could underlie cognitive decline (Ramos et al., 2006; Wang et al., 2007, 2011), whereby blockade of HCN channels can rescue age-associated declines in both working memory and associated spike rates in prefrontal cortex (Wang et al., 2007).

There is increasing data of h-current properties in human neurons as measured by transcriptomics and sag voltage recordings. Recent studies showed that HCN channel subunits are more ubiquitously expressed in pyramidal neurons across cortical layers of humans relative to rodents, with larger sag voltage in deeper layers compared to superficial layers (Kalmbach et al., 2018; Chameh et al., 2021; Rich et al., 2021). However, it is unknown if sag changes with age in human neurons as suggested by the above studies in monkeys. 
We analyzed sag voltage recordings from human neurons of younger and older subjects to check for age-associated changes in h-current and membrane properties. We then developed younger and older human pyramidal neuron models using these electrophysiological datasets, which we incorporated into microcircuit models. We simulated baseline and response activity in these human microcircuit models to characterize the effects of age-associated changes in Pyr neuron sag current on cortical processing and resting state activity.

\section{Results}

Increased sag ratio in older L5 human pyramidal neurons. We first analyzed sag ratio in human L5 Pyr neurons from the Krembil Brain Institute (KBI) and Allen Brain Institute (ABI) datasets, across subject age (Fig. 1A). Each dataset was grouped to younger ( $<50$ years) and older ( $\geq 50$ years). Sag ratio did not vary with subject age within-each group (two-sample t-test, $p>$ 0.05, Fig. 1B,C), but significantly increased in older vs. younger subjects in both datasets (twosample t-test, $p<0.05$, Cohen's $d=0.62$ and 0.65, Fig. 1D,E). This effect was present at all levels of hyperpolarization (Fig. 2D). There was no significant difference in sag ratio between neurons from males and females, in the younger group in both datasets ( $\mathrm{n}=32$ and 24 neurons from male/female in KBI, $\mathrm{n}=12$ and 32 neurons from male/female in $\mathrm{ABI}$ ).

A
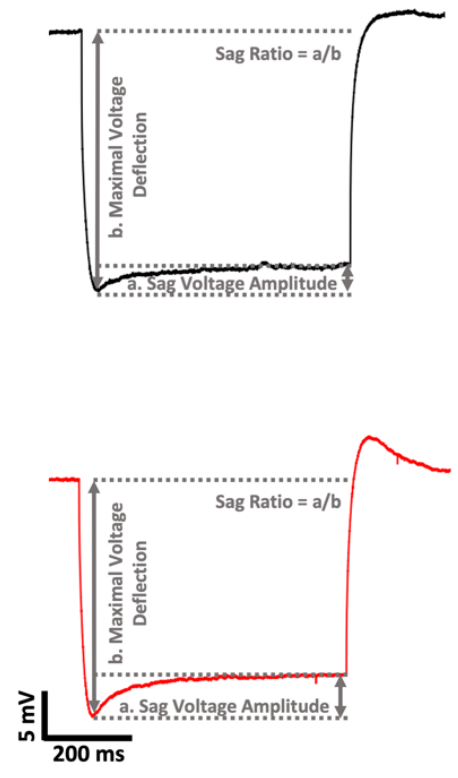

B

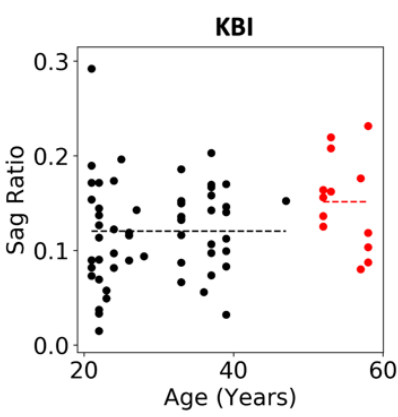

C

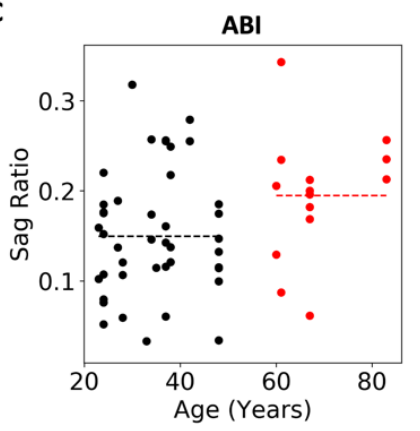

D

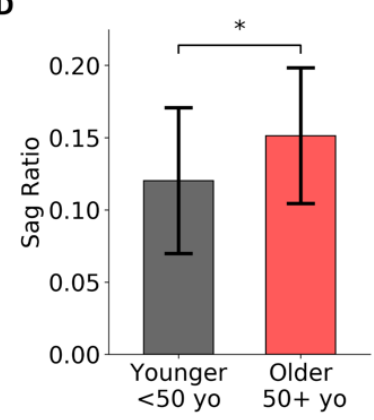

E

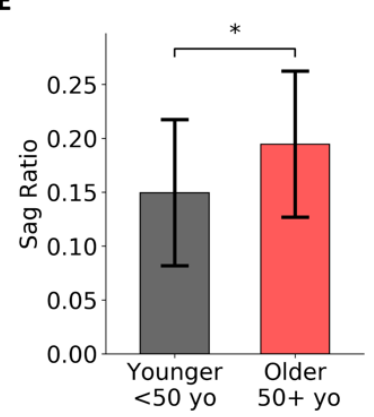

Figure 1. Increased sag ratio in older human L5 Pyr neurons. A. Example experimental voltage response to hyperpolarizing current injection $(-400 \mathrm{pA} / 600 \mathrm{~ms})$ recorded in L5 Pyr neurons from younger 
( $<50$ years, black) and older ( $\geq 50$ years, red) subjects from the KBI dataset, with an illustration of the sag voltage amplitude and ratio calculation. B-C. Sag ratio measured in L5 pyr neurons as a function of subject age, in the KBI (B) and ABI (C) datasets. Subjects were grouped into younger and older, and there was no significant relationship with age within either group. Dashed line denotes the mean sag ratio in each group. D-E. Sag ratio was significantly higher in older compared to younger subjects in both the KBI (D) and ABI (E) datasets.

We generated models for younger and older L5 Pyr neuron models using multi-objective evolutionary algorithm optimization, constrained with recordings from the KBI dataset (Fig. 2). Younger and older neuron models reproduced the experimental spiking features and hyperpolarization sag features (Fig. 2A-D; Tables S1-S2), whereby most features were within 3 standard deviations (SD) of experimental features (Tables S1-S2, see methods). We chose exemplar models for younger and older neurons (Table S4) that best reproduced the different features and had a similar fit quality between younger and older models (Fig. S1A). As seen experimentally, spiking features were similar between the younger and older neuron models (Fig. 2A-B), but the older neuron model had a larger sag ratio (Fig. 2C-D). Though the older neuron model had a more depolarized resting membrane potential (-69.8 vs. $-71.2 \mathrm{mV}$, respectively) that was in line with the experimental population mean targets (Tables S1-S2), we note that there were no significant differences in resting membrane potential between younger and older pyramidal neuron datasets. Accordingly, the older model neuron had a larger h-channel density, and this difference was seen also when comparing the set of acceptable models for younger and older neurons derived from the optimization algorithm (Fig. 2F). Due to the increased h-current density along the apical dendrites, the difference between younger and older model neurons was more pronounced in the distal apical dendrites (Fig. S1B). This difference in h-channel density was further supported by an increase in HCN1 subunit expression in human MTG L5 excitatory neurons with age ( $p<0.01$, Wilcoxon Ranked Sum test, Cohen's $d: 0.18$, log-fold increase: 0.11; Figs. 2G). 
A

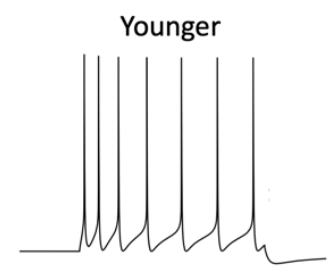

B

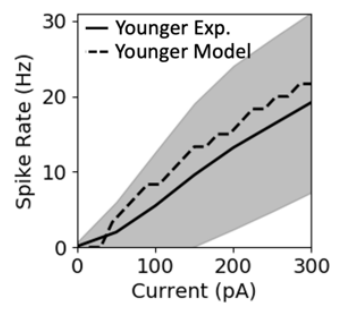

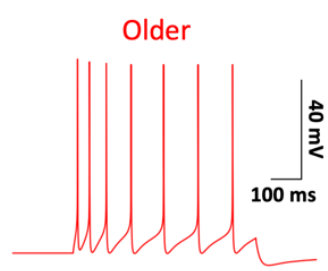

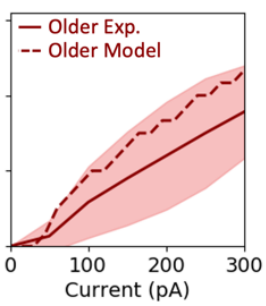

$\mathbf{E}$

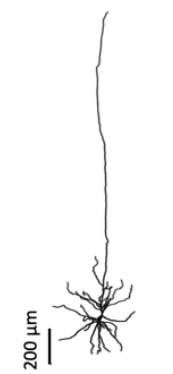

$\mathbf{F}$

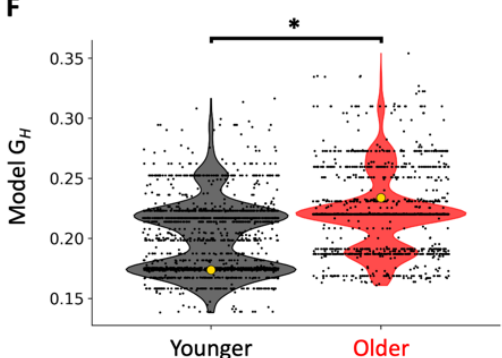

C
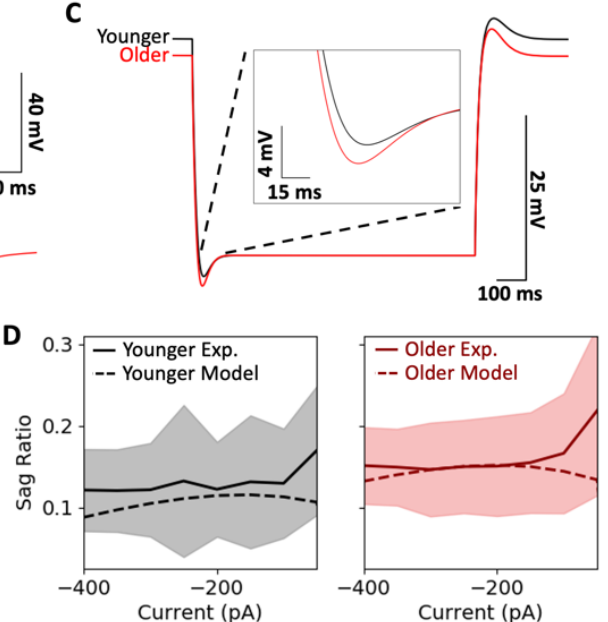

G

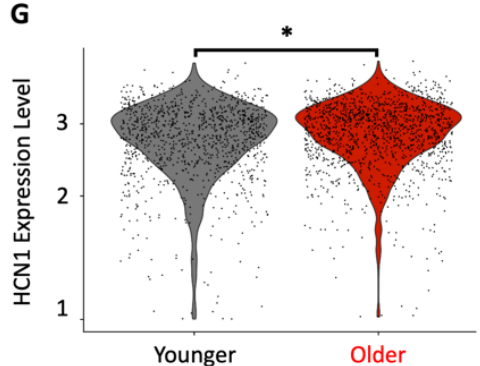

Figure 2. Human L5 Pyr neuron models capture electrical properties in younger and older data. A. Spiking output of younger and older Pyr neuron models in response to a depolarizing step current of 135 pA and duration of $600 \mathrm{~ms}$. B. Spike frequency and input current relationship for younger and older models (dashed) was within the experimental range, $<1$ SD (shaded) from the experimental mean (solid). C. Voltage response to hyperpolarizing step current of $-400 \mathrm{pA}$ and duration of $1000 \mathrm{~ms}$ in the younger and older models. Traces are aligned according to their steady-state voltages to highlight differences in sag amplitude (inset). D. Sag ratio and input current relationship for younger and older models (dashed) were within the experimental range, $<1 \mathrm{SD}$ (shaded) from the experimental mean (solid). E. Reconstructed human L5 Pyr neuron morphology, used in the younger and older neuron models. F. Violin plots of the normalized h-conductance density values for younger and older model neuron sets. Yellow circles highlight the chosen exemplar models. G. HCN1 expression levels in human L5 Pyr neurons were higher in older vs. younger ( 0.11 log-fold increase, $p<0.01$, Wilcoxon Ranked Sum test).

Dampened baseline spiking in L5 microcircuit models with older Pyr neurons. We next integrated our younger and older Pyr neuron models into microcircuit models, complete with similarly detailed models of human PV, SST, and VIP inhibitory neurons (Fig. 3A,B). We used previous models for human SST and VIP interneurons and fitted models for the human L5 PV interneuron (Tables S4). The passive and active firing features of the PV interneuron model were within the experimental variance of population data from corresponding neurons in rodents, except for AP half-width which is more dependent on the particular parameters used for the channel kinetics (Yao et al., 2022). We constrained $\mathrm{Pyr} \rightarrow \mathrm{Pyr}$ connections to reproduce experimental EPSP 
amplitudes reported in human L5 Pyr neurons (model: $0.81 \pm 0.69 \mathrm{mV}$, experimental: $0.80 \pm 0.69$ $\mathrm{mV}$; Seeman et al., 2018). The remaining connections were constrained using rodent data (Table S5).

Using the younger Pyr neuron model, we simulated human cortical L5 microcircuits of 1000 neurons with experimentally derived cortical dimensions and proportions of different neuron types (Fig. 3A, B). Each neuron received random background excitation corresponding to cortical and thalamic drive, to enable recurrent activity. We tuned the connection probabilities between neuron types and the background excitation levels to reproduce baseline firing rates previously reported for the neuron types in vivo (Fig. 3C, D). The mean firing rate for simulated Pyr neurons was $3.27 \pm 0.07 \mathrm{~Hz}, \mathrm{PV}: 15.03 \pm 0.29 \mathrm{~Hz}, \mathrm{SST}: 4.56 \pm 0.18 \mathrm{~Hz}$ and VIP: $11.36 \pm 0.32 \mathrm{~Hz}(\mathrm{n}=30$ randomized microcircuits).

We then simulated L5 microcircuit models using the older Pyr neuron model, and found a decrease in baseline Pyr neuron spike rates (Fig. 3D, E, paired-sample t-test, $p<0.001$, Cohen's $d$ : -8.7). We also tested this effect when changing the h-channel mechanism kinetics to values that have been used in previous modeling work (Hay \& Segev, 2015; Rich et al., 2021), and consistently obtained decreased baseline Pyr neuron spike rates. This decrease in baseline spike rate could be recovered and even increased by changing dendritic h-channel density and passive membrane parameter values in the older Pyr neuron model dendrites to the values used in the younger Pyr neuron model dendrites (Fig. 3E, paired-sample t-test, $p<0.001$; Cohen's $d$ : 5.4), indicating that the change in spike rate was primarily driven by differences in dendritic integration. Baseline spike rates could not be recovered by lowering the dendritic h-channel density value alone to the value used in the younger model, and even decreased the spike rates further (Fig. 3E, pairedsample t-test, $p<0.001$; Cohen's $d$ : -18.6). Thus, the age-associated decrease in spike rate was not due to changes in h-channels alone, but a combination of changes in both the depolarizing h-current and the hyperpolarizing leak current.

In line with decreased spike rates, the microcircuit models with older Pyr neurons also generated rhythmic population spiking with decreased peak frequency and power compared to the model with younger Pyr neurons (Fig. 3F). The spiking in both types of microcircuit models was in the beta frequency range, consistent with beta range frequency oscillations reported previously in local field potential recordings of L5 (Roopun et al., 2006). 
A

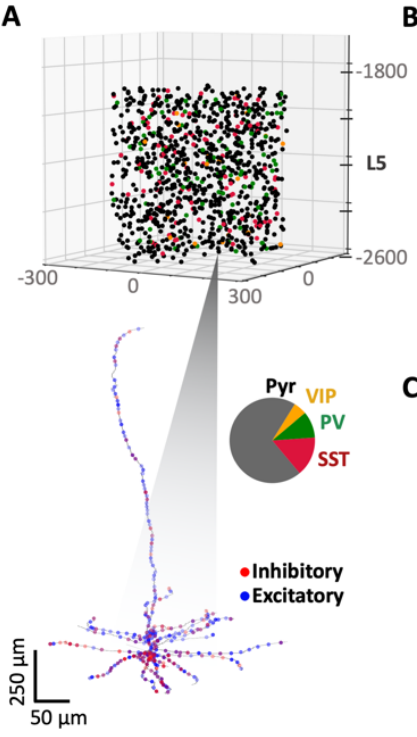

B

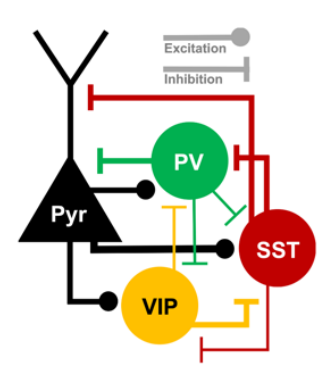

C

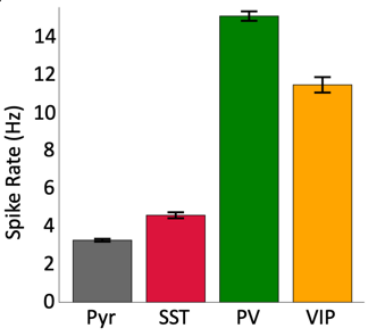

D
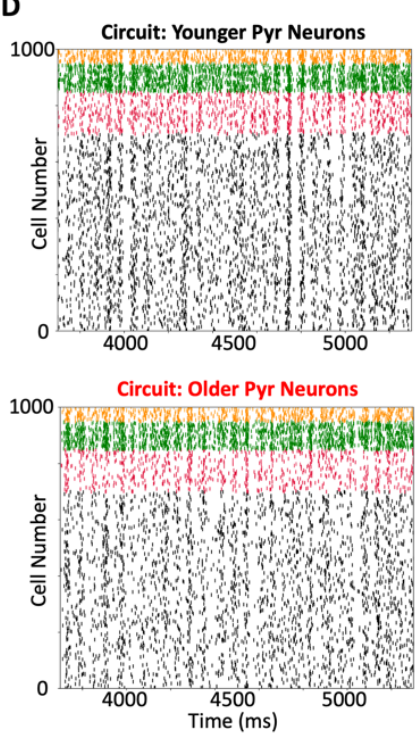

E

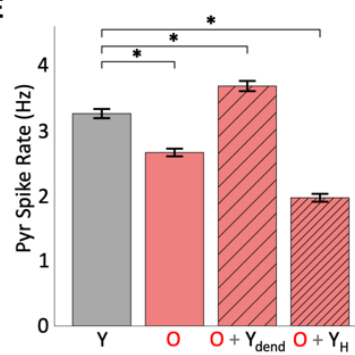

$\mathbf{F}$

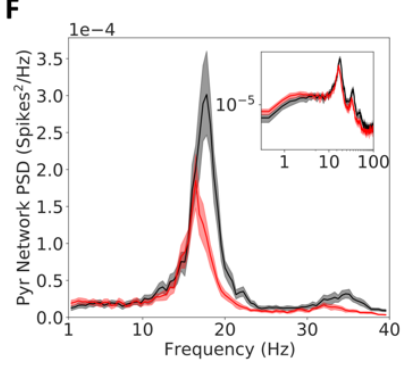

Figure 3. Dampened baseline spiking in human cortical microcircuit models with older Pyr neurons. A. The model microcircuit comprised of 1000 neurons, with the somas distributed in a $500 \times 500 \times 700 \mu \mathrm{m}^{3}$ volume along L5 (1600 to $2300 \mu \mathrm{m}$ below pia). The proportions of the different neuron types were according to the experimental transcriptomics data in human somatosensory cortical L5 (pie chart, Pyr: 70\%; SST: 15\%; PV: 10\%; VIP: 5\%). The neurons of all four types were modeled with detailed morphologies, as in Figure 2. The blue and red dots denote example excitatory and inhibitory synapses, respectively. B. Schematic diagram of the key connections between different neuron types in the microcircuit. C. Spike rates in the different neuron types reproduced experimental baseline firing rates (mean and standard deviation, $\mathrm{n}=30$ younger Pyr neuron microcircuits). D. Example raster plot of baseline spiking in the microcircuit models with younger and older Pyr neurons, color-coded according to neuron type. E. Mean Pyr neuron spike rate significantly decreased in microcircuit models with older (red; O) compared to younger (black; Y) Pyr neurons $(n=10$ microcircuits). Spike rates were recovered when changing the dendritic h-channel density and leak current parameters in the older models to that of the younger models $\left(\mathrm{O}+\mathrm{Y}_{\text {dend }}\right)$, but not when changing dendritic h-channel density alone $\left(\mathrm{O}+\mathrm{Y}_{\mathrm{H}}\right)$. F. Spikes PSD of Pyr neurons for both microcircuit models (younger, black; older, red), bootstrapped mean and 95\% confidence intervals $(n=30$ randomized microcircuits). Inset - PSD in log scale, illustrating the $1 / \mathrm{f}$ relationship between power and log frequency.

To validate our model prediction that baseline spike rates of older human L5 pyramidal neuron microcircuits are reduced, we analyzed multi-electrode array (MEA) extracellular recordings across all layers of human cortical slices (Fig. 4A). These slices exhibited baseline firing rates on the same order of magnitude as the simulated circuit (Fig. 4B-C). In the L5 areas of the slice, spike rates of broad-spiking single units (putative Pyr neurons) in older subjects were decreased compared to younger subjects (two-sample t-test, $p<0.05$; younger: mean $=1.09 \mathrm{~Hz}$, 95\% CI $=0.87-1.30 \mathrm{~Hz}$; older: mean $=0.49 \mathrm{~Hz}, 95 \% \mathrm{CI}=0.30-0.64 \mathrm{~Hz}$; Cohen's $d:-0.27$, respectively; $\mathrm{n}=401$ units from 14 younger subjects, $\mathrm{n}=51$ units from 4 older subjects; Fig. 4BC, right). Similarly, spike rates in L2/3 areas of the slice were also decreased in older subjects 
compared to younger subjects (two-sample t-test, $p<0.05$; younger: mean $=1.27 \mathrm{~Hz}, 95 \% \mathrm{CI}=$ $0.97-1.52 \mathrm{~Hz}$; older: mean $=0.46 \mathrm{~Hz}, 95 \% \mathrm{CI}=0.31-0.57 \mathrm{~Hz}$; Cohen's $d:-0.37 ; \mathrm{n}=376$ units from 14 younger subjects, $n=148$ units from 5 older subjects; Fig. 4B-C, left). Spike rates did not change significantly in L4 broad-spiking units with age, though this may be partly due to the presence of stellate neurons in L4 (Fig. 4B-C, middle). Compared to the $18.3 \%$ decrease in spike rates in our models with older L5 Pyr neurons due to sag current changes alone, the L5 MEA data from older subjects showed a larger 55\% decrease, but with a smaller effect size (Cohen's $d:-8.7$ vs -0.27 , respectively) due to the larger variability in the data.

A

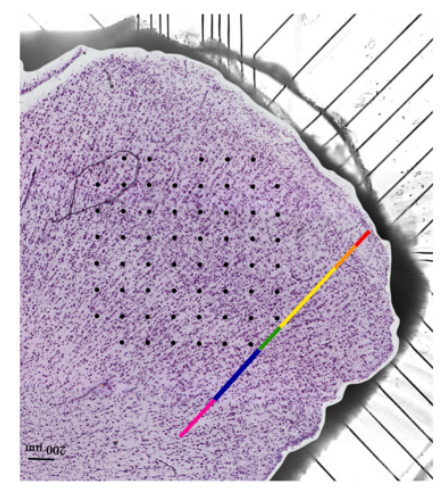

B

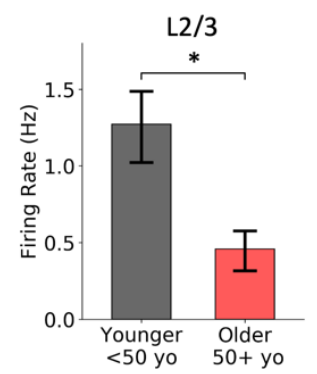

C

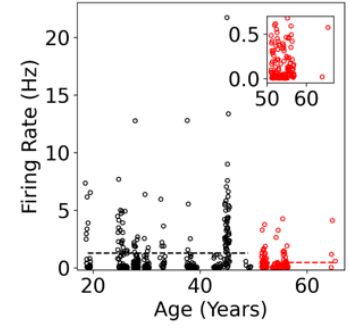

L4
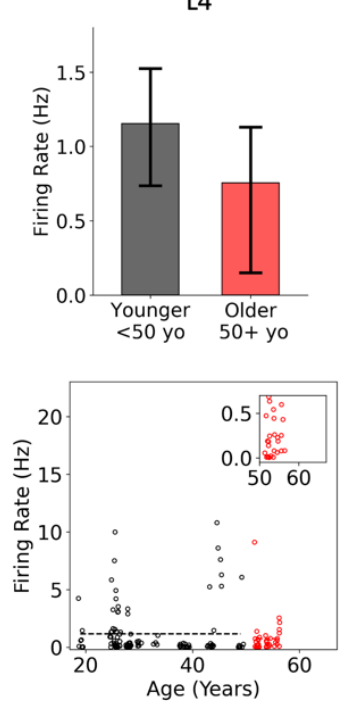

L5
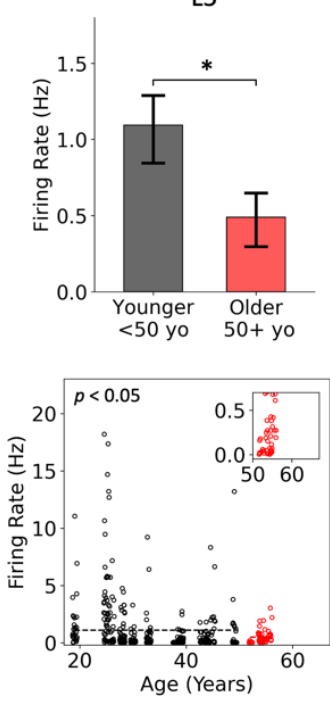

Figure 4. MEA recordings reveal dampened microcircuit spiking in older human cortical L5 and L2/3 Pyr neurons. A. Human cortical tissue (500 $\mu \mathrm{m}$ thickness; $5 \mathrm{X}$ lens) in a 60-channel MEA. Black dots denote electrode locations and the color bar denotes layers 1 (red, upper right) through 6 (pink, lower left) of the human cortical slice which was Nissl stained after the recording and overlaid on the image of slice during the recording. B. Bootstrapped mean firing rates and $95 \%$ confidence intervals obtained from extracellular recordings by planar MEAs across layers of cortical slices. Firing rate did not change in L4 with age but was significantly decreased in older L2/3 and L5 Pyr neurons. C. Firing rate measured in L2/3, L4, and L5 neurons as a function of subject age. Dashed line denotes the mean firing rate in each group. Inset plots show expanded axes for the older firing rate data.

Increased sag current has minimal effect on microcircuit stimulus response. To assess the effect of sag current changes on microcircuit response, we examined tuning selectivity as a typical and generalizable example of cortical processing. We simulated orientation-selectivity in our models by applying thalamic inputs to orientation-selective neurons, with tuning curves as reported experimentally from monkey somatosensory cortex (Fig. 5A, B). We simulated different 
presentations of $85^{\circ}$ or $95^{\circ}$, representing a small orientation difference, and thus a challenging discrimination task due to similar levels of circuit activation (Fig. 5B). Stimulus inputs included randomized additive noise and were applied to a microcircuit with fixed connectivity but random background input (Fig. 5C-E). Across 80 stimulus presentations for each angle, response rates of Pyr neurons were reduced in microcircuits with older Pyr neurons (paired-sample t-test, $p<0.05$; younger: $10.39 \pm 0.66 \mathrm{~Hz}$; older: $10.19 \pm 0.64 \mathrm{~Hz}$; Cohen's $d$ : -0.3 , Fig. 5F), although to a much lesser degree than the decrease in baseline rates (paired-sample t-test, $p<0.001$; younger: $3.25 \pm$ $0.03 \mathrm{~Hz}$; older: $2.64 \pm 0.03 \mathrm{~Hz}$; Cohen's $d$ : -19.8). The larger dampening of baseline activity compared to response thus imposed an increased signal-to-noise ratio (SNR) in microcircuits with older Pyr neurons compared to those with younger Pyr neurons (Fig. 5G; paired-sample t-test, $p$ $<0.001$; younger: $3.06 \pm 0.22$; older: $3.68 \pm 0.27$; Cohen's $d: 2.5)$. Pyr neuron sag current changes thus did not account for reduced response rates and decreased SNR seen in aging, indicating the involvement of other mechanisms.

A

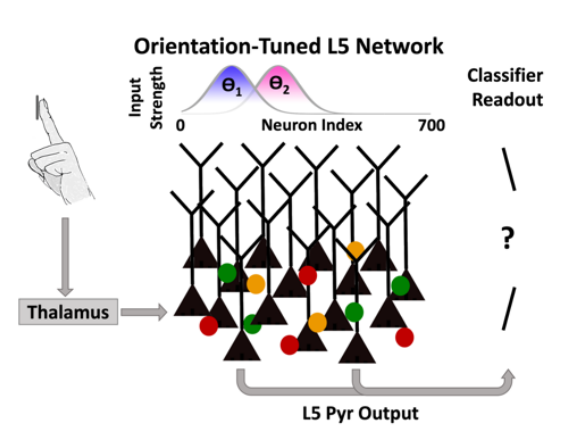

$\mathbf{F}$

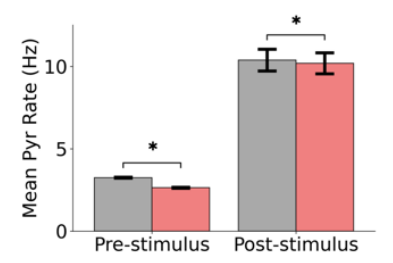

B

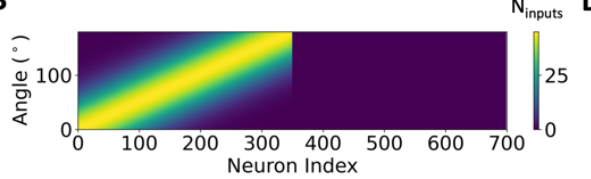

C

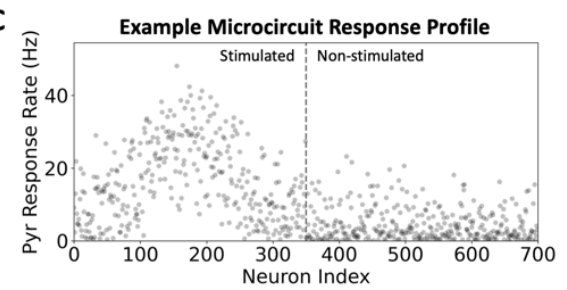

H
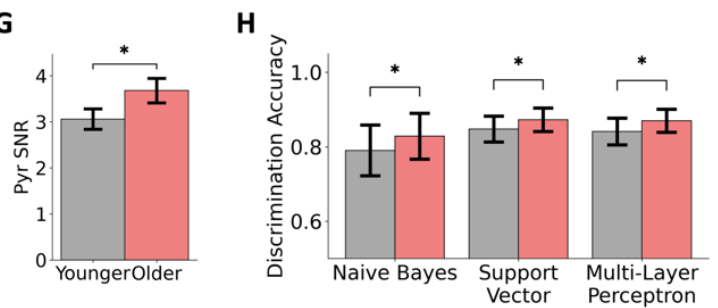

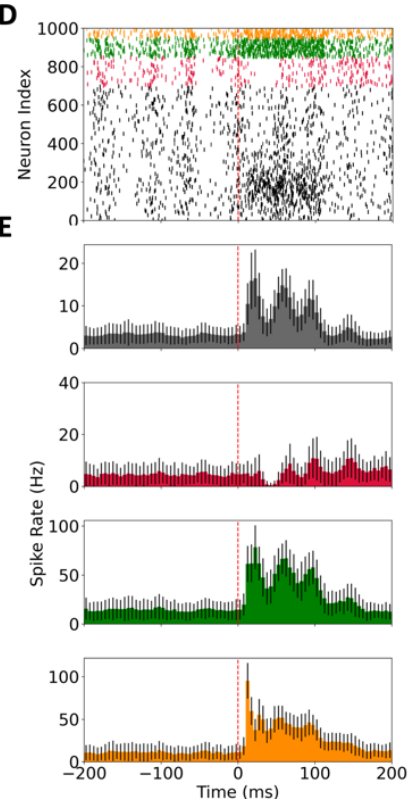

Figure 5. Increased sag current in older Pyr neurons has minimal effects on stimulus response. A. Stimulus response simulation paradigm, where orientation-selective neurons in the L5 microcircuit received thalamic inputs corresponding to tactile edges of different orientations (e.g. $\Theta_{1}, \Theta_{2}$ ). The Gaussian distributions show the activation of different subgroups of neurons in response to different orientations. L5 Pyr neuron output responses to randomized stimulus presentations for each stimulus orientation $\left(85^{\circ}\right.$ or $95^{\circ}$ ) were inputted into classifiers to assess discrimination accuracy. B. Orientation-tuning of Pyr neurons in the microcircuit, relating the number of thalamic inputs to each tactile edge orientation. $\mathbf{C}$. Example average response rate for neurons in a microcircuit with younger Pyr neurons during $(\mathrm{n}=80)$ presentations of the $85^{\circ}$ stimulus. D. Example raster plot of simulated spike response in a microcircuit model with younger Pyr neurons during presentation of an $85^{\circ}$ stimulus. Dashed line denotes the stimulus time. E. 
Average peri-stimulus time histograms during $(n=80) 85^{\circ}$ stimulus presentations in the microcircuit model with younger Pyr neurons. F. Baseline and response spike rates to $85^{\circ}$ inputs (mean \pm SD) in both microcircuit models ( $n=80$ stimulus presentations each). G. Moderately increased SNR in cortical microcircuit models with older Pyr neurons compared to those with younger Pyr neurons. H. Moderately increased stimulus classification accuracy using readout from cortical microcircuits with older Pyr neurons compared to those with younger Pyr neurons, across different classifier types $(n=300$ permutations of the train/test datasets).

We next determined the implications of SNR on the accuracy of discrimination of stimulus orientations using readout from microcircuits with older vs. younger Pyr neurons. The readout comprised of Pyr neuron output metrics that served as feature inputs into different classifier types (Fig. 5H). Across all classifiers, we found a moderate increase in discrimination accuracy when using output features from microcircuit models with older Pyr neurons compared to the microcircuit models with younger Pyr neurons (Fig. 5H; two-sample t-test, $p<0.05$; Cohen's $d$ values: Naïve Bayes $=0.6$; Support Vector $=0.7$; Multi-Layer Perceptron $=0.9$ ). The increase in microcircuit SNR mediated by the changes in sag current mechanisms in older L5 Pyr neurons therefore led to some improvement in decoding signals from the neuronal activity readout. We also ran similar tests using spike rate or maximum PSP amplitude as alternative feature inputs, and these generated results that were consistent with our results when using area under the curve. We next examined Pyr neuron responses to apical dendrite inputs, as well as lower stimulus magnitudes (Fig. 6A). In all cases, changes to response rates were minimal (Fig. 6B; Cohen's $d$, paired-sample t-test: low basal $=-0.8, p<0.01$; low apical $=-1.3, p<0.001$; basal $=-0.6, p<0.01$; apical $=0.3, p>0.05)$ and SNR was higher in microcircuit models with older Pyr neurons (Fig. 6C; paired-sample t-test, $p<0.001$, Cohen's $d$ : low basal $=1.6$; low apical $=1.4$; basal $=2.0$; apical =2.9). These effects on SNR were smaller for responses to weaker inputs $(\mathbf{F i g} \mathbf{6 C})$ due to larger reductions in response rates with age in this condition (Fig 6B). 
A
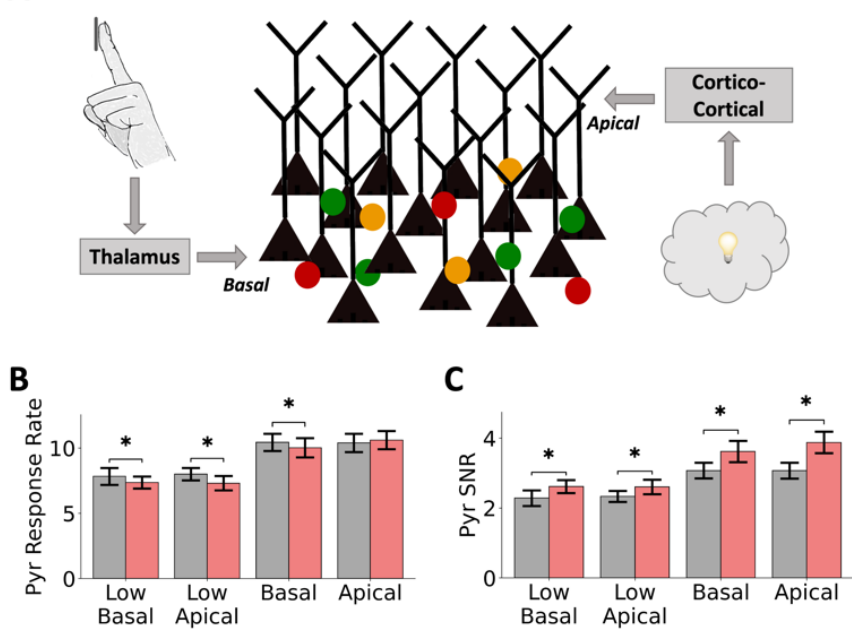

Figure 6. Age-associated changes are robust across stimulus location and amplitude. A. Stimulus response simulation paradigm, where the Pyr cells are stimulated by either thalamic inputs (representing tactile edges) onto their basal dendrites, or cortical inputs (representing associative information) onto their apical dendrites. B. Minimal effects on response rates in cortical microcircuit models with older Pyr neurons compared to those with younger Pyr neurons $(n=30$ stimulus presentations) for low/high input onto basal/apical dendrites. C. Increased Pyr neuron SNR in cortical microcircuit models with older Pyr neurons compared to those with younger Pyr neurons.

As the differences in excitability between younger and older Pyr neuron models were primarily driven by changes in dendritic h-channel density and membrane leak current parameters (Fig. 3E), we examined excitatory (Fig. 7A) and inhibitory (Fig. 7C,E) PSP summation in the models to characterize possible reasons for the dampened firing rates in microcircuits with older Pyr neurons. The peak excitatory (Fig. 7B, top) and inhibitory (Fig. 7D,F, top) PSPs (from Pyr and SST/PV connections, respectively) in the older neuron model were more depolarized than in the younger neuron model, due to the depolarized resting membrane potential resulting from larger h-channel density. In addition, the inhibitory PSP amplitude of the PV $\rightarrow$ Pyr connection increased considerably (paired-sample t-test, $p<0.05$, Cohen's $d: 5.4$ ). While we also observed decreased $\mathrm{Pyr} \rightarrow \operatorname{Pyr}$ (paired-sample t-test, $p<0.05$, Cohen's $d:-0.6$ ) and increased SST $\rightarrow$ Pyr (paired-sample t-test, $p<0.05$, Cohen's $d$ : 0.2) amplitudes, these effects were small. When current-compensated to equalize resting membrane potential between the models, excitatory (Fig. 7B, bottom) and inhibitory (Fig. 7D,F, bottom) PSP summation in the older neuron model were dampened compared to the younger neuron model (paired-sample t-test, $p<0.05$, Cohen's $d: \mathrm{Pyr} \rightarrow \mathrm{Pyr}=$ $1.9, \mathrm{SST} \rightarrow \mathrm{Pyr}=-1.7, \mathrm{PV} \rightarrow \mathrm{Pyr}=-1.5)$. Across all connection types, the PSP time course was shortened in the older neuron model, reflecting the larger h-current effects. Although the net effect 
on IPSP and EPSP summation in the microcircuit depends on the circuit state and neuronal membrane potential, the dampened summation in older Pyr neurons for a given membrane potential would generally account for the reduced firing rates.
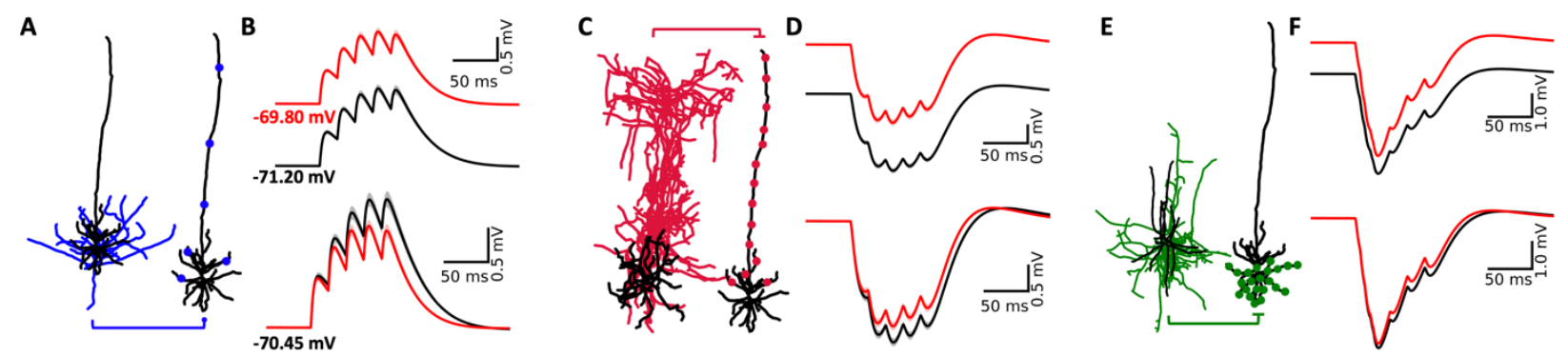

Figure 7. Synaptic input summation in younger and older neuron models. A. $\mathrm{Pyr} \rightarrow \mathrm{Pyr}$ connections tested for EPSP summation. Circles mark synaptic contacts onto the post-synaptic Pyr neuron. B. Top traces show EPSP summation ( $50 \mathrm{~Hz}$ train of 5 step current pulses) in the younger (black) and older (red) neuron models (20 connections, 20 trials/connection), bootstrapped mean and 95\% confidence intervals. Bottom traces show the same as the top traces, but when resting membrane potential is equalized between younger and older neuron models by current compensation. C-F. Same as A-B but for IPSP summation of SST $\rightarrow$ Pyr (C-D) and PV $\rightarrow$ Pyr (E-F) connection types.

\section{Discussion}

Here we found an increase in sag ratio of human cortical L5 pyramidal neurons with age, which led to reduced baseline activity and noise during response in simulated microcircuits. Analysis of extracellular recordings using MEA across layers ex vivo further confirmed a decrease in L5 pyramidal neuron spike rates in cortical tissue from older individuals. We demonstrated the link mechanistically using novel detailed models of human L5 microcircuits that integrated human cellular and circuit data. Our simulations show that although changes in Pyr neuron sag current mechanisms did not play a role in age-dependent reduced response rates and discrimination acuity, the sag current changes alone can to account for the reduced baseline spike rates seen in aging. Our models thus reproduce age-dependent changes in cortical resting state activity, and may serve to further study their implications and clinical relevance in aging.

The reduced baseline and response rates in our microcircuit models in aging, and the involvement of h-currents, agree with previous studies in monkeys (Wang et al., 2007, 2011). Two key differences in the above studies, however, were a larger reduction in response rates accompanied with cognitive deficits in working memory, and a global suppression of h-channels rescuing both response spike rates and working memory deficits. Our findings thus indicate that the effects of global h-channel suppression on circuit spiking in monkeys likely go beyond the 
impact in Pyr neurons that we have modelled here. These discrepancies may be due to multiregional effects, or the involvement of age-changes in other mechanisms as indicated by our simulations of response and discrimination acuity. Possible additional age-associated mechanisms through which reduction in response rates and cortical processing quality could occur include reduced SST and VIP inhibition (Mohan et al., 2018), neuronal atrophy (Prevot et al., 2021), homeostatic reductions in tonic inhibition (Chen et al., 2010; Prevot et al., 2021), and reduced NMDA/AMPA ratios (Pegasiou et al., 2020). There may also be sag current changes in other neuron types such as SST interneurons, which exhibit large sag current like Pyr neurons, but for which aging data is not yet available. The possible involvement of the above mechanisms may also depend on associative distal dendritic inputs onto L5 pyramidal neurons, which we demonstrated can exhibit similar effects on baseline and response rates due to increased sagcurrent in the microcircuit models with older Pyr neurons. Analysis of ex vivo MEA data also validated our predictions of decreased L5 pyramidal neuron spiking in cortical microcircuits of older individuals. While the decrease in microcircuit spiking was comparable to the changes seen in our microcircuit models involving sag current change alone, differences in the magnitude of the decrease could be attributed to the involvement of other mechanisms mentioned above. Whereas in the current study we have assessed the functional impact of Pyr neuron changes with age, it will be of interest in future studies to explore how additional diverse mechanisms refine our results to explain the rest of the effects seen in aging.

We studied the effect of the age-dependent cellular changes on sensory cortical processing, since the underlying microcircuits are well-studied (Yu et al., 2019; Thakur et al., 2006; Bensmaia et al., 2008) and since sensory processing worsens with age. Previous studies showed that tactile acuity decreases with age (Legge et al., 2008, 2019), and perceptual speeds and reaction times are slower (Bucur \& Madden, 2010). Furthermore, the simulation of cortical response to sensory stimuli served to study a prototypical cortical microcircuit processing to bottom-up inputs together with recurrent inputs from the microcircuit neurons onto Pyr neuron basal and apical dendrites (Hay \& Segev, 2015). The consistent age effects we saw when simulating the processing of apical inputs, which would correspond to top-down connections from higher-order cortical areas, indicate that the results should generalize to other types of cortical processing relevant to aging.

The age-dependent reduction in microcircuit spike rates involved a combination of dendritic mechanisms underlying the increased sag ratio, including h- and leak-currents. Despite 
the older models being more depolarized, circuit spiking was reduced due to increased h-current dampening synaptic summation, in agreement with previous studies (Williams \& Stuart, 2000; Song \& Moyer, 2017), and due to a reduced excitatory driving force and an increased inhibitory driving force. The increase in h-current estimated from our models and electrophysiology was further supported by analysis of HCN1 expression in human Pyr neurons. HCN channel subunits are expressed ubiquitously across layers of human cortex, more than in rodents, further suggesting their importance in human Pyr neurons (Kalmbach et al., 2018). Although we have studied the effect of Pyr neuron sag current, it remains unknown whether there are also age-associated changes in interneuron sag current, which would modulate cortical firing rates. SST, PV, and VIP expressing interneurons have all demonstrated h-currents in electrophysiological recordings (Albertson et al., 2017; Prönneke et al., 2015; Roth \& Hu, 2020). When human interneuron electrophysiology data becomes more abundant, a similar approach could be used to estimate ageassociated changes and integrate them into the models to better understand the microcircuit changes in aging.

Our microcircuit models capture several aspects of biological L5 circuits, including human cell type proportions, human neuronal intrinsic properties, in vivo spike rates, and stimulus response rates. When dendritic properties of human L5 Pyr neurons become better understood (Beaulieu-Laroche et al., 2018; Gidon et al., 2020), it will be interesting to investigate the possible contribution of dendritic features such as action potential backpropagation and calcium spikes to age changes in dendritic h-currents, which could amplify the effect on network response rates (Hay \& Segev, 2015). The Pyr neuron models in our study were constrained with both morphological and spiking features of intratelencephalic-projecting-like neurons, in line with the higher proportion of these neurons in human MTG relative to extratelencephalic-projecting Pyr neurons (Kalmbach et al., 2021; Chameh et al., 2021). Our circuit models spiking exhibited beta range frequency oscillations, which have been reported in local field potential recordings of L5 (Roopun et al., 2006; Halgren et al., 2018). The slowing of the peak frequency and reduced power with age in our models may also bear similar mechanisms to the slowing and decreased power of alpha rhythms with age that have been reported in EEG datasets (Choi et al., 2019; Donoghue et al., 2020). Future models involving also the superficial cortical layers would enable linking the agedependent changes to the associated EEG signals (Hagen et al., 2018), and relating them to 
experimental recordings (Choi et al., 2019; Donoghue et al., 2020). All models and simulation code will be available openly online upon publication.

\section{Methods}

Electrophysiology Data. We used whole-cell recordings of human middle temporal gyrus layer 5 (L5) pyr neurons reported previously in datasets from Krembil Research Institute (KBI; Chameh et al., 2021; Younger: $\mathrm{n}=56$ neurons, 32 neurons from male subjects, 24 neurons from female subjects; Older: $\mathrm{n}=13$ neurons, 1 neuron from male subjects, 12 neurons from female subjects) and Allen Brain Institute (ABI; Gouwens et al., 2018; Younger: $\mathrm{n}=44$ neurons, 12 neurons from male subjects, 32 neurons from female subjects; Older: $\mathrm{n}=14$ neurons, 14 neurons from male subjects, 0 neurons from female subjects). Subject age in the KBI and ABI datasets ranged between 21 - 58 years, and 23 - 83 years, respectively. We grouped the subjects into younger ( $<50$ years) and older ( $\geq 50$ years) in line with previous studies (Davis et al., 1990; Bucur \& Madden, 2010; McIntosh et al., 2014). For sag ratio calculations across both datasets, the largest hyperpolarization step was used (-400 pA for KBI; -110 pA for ABI). We also used intracellular recordings of human putative L5 parvalbumin (PV, neuron ID: 528687520) interneurons available from ABI (Gouwens et al., 2018). We used reconstructed human neuron morphologies from ABI for the L5 Pyr neuron (Neuron ID: 562381210; donor age $=34$ years) and the PV interneuron. The L5 pyramidal neuron morphology that we chose was a typical intra-telencephalic-projecting (IT)-like, which is the most abundant Pyr neuron type in human cortical layer 5 (Kalmbach et al., 2021; Chameh et al., 2021). We used depolarizing and hyperpolarizing current steps for neuron model optimization (Tables S1-S3), whereby Pyr neuron data was from the KBI dataset because it included a larger range of hyperpolarizing and depolarizing step current amplitudes (i.e., as low as $-400 \mathrm{pA}$ and as high as $300 \mathrm{pA}$ ). Three depolarizing supra-threshold steps were used for fitting spiking features at low, medium and high firing rates. The hyperpolarizing step currents were used for fitting passive and sag features.

We also used extracellular recordings using MEA from resected human middle temporal gyrus slices (500 $\mu \mathrm{m}$ thickness) maintained active in carbogenated $\left(95 \% \mathrm{O}_{2}, 5 \% \mathrm{CO}_{2}\right)$ artificial cerebral spinal fluid (Florez et al., 2015). The electrodes were registered on the slices to identify the anatomical location of the single units recorded. Putative cell types were identified based on 
waveform features, mainly the peak to trough latency of the average spike waveform (Barthó et al., 2004). We used the waveform feature to cluster units into broad spiking and narrow spiking units. Subsequent spike rate analyses were performed on broad-spiking units (putative pyramidal neurons). Subject age ranged between $19-65$ years and were grouped subjects into younger $(<50$ years) and older ( $\geq 50$ years) groups (Younger: $n=376$ L2/3, 119 L4, and 401 L5 units from 17 subjects; Older: $\mathrm{n}=148$ L2/3, 37 L4, and 51 L5 units from 5 subjects).

Human L5 Microcircuit Models. We simulated L5 microcircuits comprised of 1000 neurons distributed along a layer 5 volume $\left(500 \times 500 \times 700 \mu \mathrm{m}^{3}\right.$, 1600 to $2300 \mu \mathrm{m}$ below pia; Mohan et al., 2015) using NEURON (Carnevale \& Hines, 2006) and LFPy (Hagen et al., 2018). In addition to Pyr and PV neurons, the microcircuit models included somatostatin (SST) and vasoactive intestinal peptide (VIP) interneurons, using previous human L2/3 neuron models (Yao et al., 2022), because there was no human data for these interneurons from L5. Neurons of a given type had the same model (morphology and biophysical properties) but differed in the randomization of their synaptic connectivity and background input. The proportions of the four neuron types in the microcircuit were: 70\% Pyr, 15\% SST, 10\% PV, and 5\% VIP, approximated using L5 RNA-seq data from the Allen Human Brain Atlas (Hodge et al., 2019).

Human Neuron Models. We developed multi-compartmental models for the Pyr and PV neurons using the BluePyOpt Python module to perform multi-objective optimizations (Van Geit et al., 2016). We used a set of ion channel mechanisms taken from previously published models (Hay et al., 2011, 2013; Yao et al., 2022). The models were fit in one step where all passive and active parameters and features were optimized simultaneously (Tables S1-S3). We set the following parameters: $R_{a}=100 \Omega \mathrm{cm}, E_{N a}=50 \mathrm{mV}, E_{K}=-85 \mathrm{mV}$, and CaDynamics gamma $=0.0005$ (Hay et al., 2011). We also set the somatic and axonal NaT kinetics to Sshift $_{m}=0$, Vshift $_{h}=10$, Slope $_{m}=$ 9, and Slope $_{h}=6$. The specific membrane capacitance $\left(c_{m}\right)$ was $0.9 \mu \mathrm{F} / \mathrm{cm}^{2}$ for the Pyr models, and $2 \mu \mathrm{F} / \mathrm{cm}^{2}$ for the PV model to reproduce membrane time constants (possibly due to errors in PV dendritic diameter estimation; Table S4). Model optimization was run using parallel computing clusters [Neuroscience Gateway (NSG; Sivagnanam et al., 2013): 400 processors with a population size of 400, across 300 generations and an approximate total runtime of 5 hours]. Additionally, $\bar{g}_{H}$ was distributed uniformly across all dendritic sections of the PV model (similarly to the SST and 
VIP models). For Pyr neurons, we have adapted an exponential function used in previous models (Hay et al., 2011) to an equivalent sigmoidal function allowing saturation at a certain distance from soma, so that $\bar{g}_{H}$ increased with distance from soma along the basal and apical dendrites as follows:

$$
\bar{g}_{H}=\bar{g}_{H, \text { soma }}(0.5+(24 /(1+\exp ((X-950) /-285))))
$$

where $X$ is the distance from soma in $\mu \mathrm{m}$. Model performance in reproducing the electrophysiology features was assessed in terms of standard deviation from the experimental mean. For Pyr models we used the statistics over the set of recorded neurons from the KBI dataset, and for the PV model (where data from only a single human neuron was available), we used the population variance from the rodent literature (Zurita et al., 2018).

Synaptic Connectivity Models. We used previous models of NMDA/AMPA excitatory and $\mathrm{GABA}_{\mathrm{A}}$ inhibitory synapses, that incorporated presynaptic short-term plasticity parameters for vesicle-usage, facilitation, and depression, as well as separate time constant parameters for the AMPA and NMDA -mediated components of excitatory synapses (Fuhrmann et al., 2002; Hay et al., 2013; Mäki-Marttunen et al., 2019). We set the same time constant parameters for all connection types $\left(\tau_{\text {rise }, N M D A}=2 \mathrm{~ms} ; \tau_{\text {decay }, N M D A}=65 \mathrm{~ms} ; \tau_{\text {rise,AMPA }}=0.3 \mathrm{~ms} ; \tau_{\text {decay,AMPA }}=3 \mathrm{~ms}\right.$; $\left.\tau_{\text {rise }, G A B A}=1 \mathrm{~ms} ; \tau_{\text {decay }, G A B A}=10 \mathrm{~ms}\right)$, as well as the reversal potential values $\left(E_{\text {exc }}=0 \mathrm{mV} ; E_{\text {inh }}=\right.$ $80 \mathrm{mV})$.

For $\mathrm{Pyr} \rightarrow \mathrm{Pyr}$ connections, we fitted the synaptic conductance and vesicle-usage parameters using the human experimental literature (Seeman et al., 2018). We simulated the experimental conditions (e.g., chloride reversal potential, and holding currents) and adjusted the conductance and vesicle-usage parameters to achieve the target postsynaptic (PSP) amplitudes and failure rates on average across 50 randomizations of synaptic locations and events. For the conductance and vesicle-usage parameters of all other connection types, as well as the depression, facilitation, and numbers of synaptic contacts $\left(N_{\text {syns }}\right)$ for all connections, we used values reported by the Blue Brain Project (Ramaswamy et al., 2015). For SST $\rightarrow$ Pyr connections, we used a higher conductance, consistent with SST $\rightarrow$ Pyr conductance of human synaptic connections in L2/3 (Yao et al., 2022). The synaptic parameters of the different connections are summarized in Table S5. Specific synaptic locations onto Pyr neurons were dependent on the connection type, where $\mathrm{Pyr} \rightarrow \mathrm{Pyr}$ synapses were placed on both basal and apical dendritic compartments, PV $\rightarrow \mathrm{Pyr}$ connections were placed on basal dendritic compartments, and SST $\rightarrow$ Pyr connections were placed 
on apical dendritic compartments. Apart from these specifications, synapse locations were chosen randomly from a uniform distribution.

We initially set unidirectional connection probability $\left(p_{c o n}\right)$ according to the rodent literature (Blue Brain Project and $\mathrm{ABI}$ ), except for $\mathrm{Pyr} \rightarrow \mathrm{Pyr}$ connections, where $p_{\text {con }}$ was set to $12 \%$ according to human literature (Seeman et al., 2018). We then adjusted connection probabilities guided by the reported experimental ranges to reproduce the intrinsic activity, which required a decrease of the $\mathrm{Pyr} \rightarrow \mathrm{Pyr}$ connection probability down to $9 \%$. The connection probabilities in the microcircuit are summarized in Table S5.

Modeling Microcircuit Intrinsic Activity. We constrained the microcircuit to generate baseline spike rates as reported for different L5 neuron types in rodents in vivo (range, median; Pyr: 0.4 11.5 Hz, 2.6 Hz; PV: 4.6 - 22.0 Hz, 17.2 Hz; SST: 0.5 - 6.9 Hz, 1.7 Hz; VIP: 8.5 - 21.0 Hz, 11.1 $\mathrm{Hz}$; $\mathrm{Yu}$ et al., 2019), by adjusting the $p_{\text {con }}$ values guided by the reported experimental ranges for all connection types, and by adjusting the background input (see below). We calculated the simulated rates across non-silent neurons $(>0.2 \mathrm{~Hz})$ over 4.5 seconds of baseline simulation. Average rates were then computed across 30 randomized microcircuits. The microcircuit received random uncorrelated background excitatory input using Ornstein-Uhlenbeck (OU) point processes (Destexhe et al., 2001), placed at halfway the length of each dendritic arbor to ensure similar levels of inputs along each dendritic path. For the Pyr models, we placed 5 additional OU processes along the apical trunk at $10 \%, 30 \%, 50 \%, 70 \%$, and $90 \%$ of the apical dendritic length. We set the base excitatory $\mathrm{OU}$ conductance to the following: $\mathrm{Pyr}=76 \mathrm{pS}$; SST $=32 \mathrm{pS}$; PV $=1545 \mathrm{pS}$ (due to higher rheobase); VIP $=75 \mathrm{pS}$. We did not use an inhibitory OU conductance since the model microcircuit provided sufficient inhibition. Furthermore, we scaled the OU conductance values to increase with distance from soma by the multiplying them with the exponent of the relative distance from soma (ranging from 0 to 1$): \bar{g}_{O U}=\bar{g} \times \exp \left(X_{\text {relative }}\right)$.

Tonic Inhibition. We modelled tonic inhibition using a previous model for outward rectifying tonic inhibition (Bryson et al., 2020). We used previous estimates of tonic inhibition conductance ( $G_{\text {tonic }}$; uniformly across all somatic, basal, and apical compartments) from a human L2/3 microcircuit model ( $G_{\text {tonic }}: 0.938 \mathrm{mS} / \mathrm{cm}^{2}$ for all neuron types; Yao et al., 2022), since total tonic inhibition current in L2/3 Pyr neurons was similar to estimates of the total tonic inhibitory current 
measured in L5/6 neurons (Scimemi et al., 2006). As well, it has previously been shown that Pyr neurons and interneurons in L5/6 have similar levels of tonic inhibition after correcting for cell capacitance (Scimemi et al., 2006).

Modeling Microcircuit Response Activity. We modelled Pyr neuron responses according to previous studies of somatosensory cortex in awake monkeys during tactile stimulation of the finger by indentation with an edge at varying orientations (Thakur et al., 2006; Bensmaia et al., 2008). We constrained neuronal tuning curve maximal rates in the range of 10 to $120 \mathrm{~Hz}$ (Thakur et al., 2006; Bensmaia et al., 2008), and tuning curve half-width in the range of $34^{\circ}$ to $49^{\circ}$ (Bensmaia et al., 2008). We applied orientation-selectivity to 50\% of the Pyr neurons (Bensmaia et al., 2008), as well as the PV and VIP neurons (Sermet et al., 2019). Pyr basal dendrites, PV neurons, and VIP neurons were stimulated using excitatory AMPA/NMDA synapses with the same synaptic dynamics and conductance as the intra-cortical excitatory synapses above. We injected the thalamic input synapses with noisy artificial presynaptic inputs spiking at $100 \mathrm{~Hz}$, with inter-spike intervals randomly sampled from a negative exponential distribution, over the course of a $100 \mathrm{~ms}$ window, in line with the spiking of ventral posterior nucleus neurons (which provide inputs to somatosensory cortex) recorded in awake monkeys during finger indentations (Song \& Semework, 2015). Average response rates were calculated over the $100 \mathrm{~ms}$ window following stimulus onset. We did not tightly constrain the response spike rates of the interneurons due to a lack of experimental data, but rather constrained the PV and VIP neurons to be activated, while SST neuron response rates were either unchanged or silenced (Gentet et al., 2012; Yu et al., 2019; Sermet et al., 2019; Muñoz et al., 2017).

Neurons in the microcircuit had different preferred orientations, so that angles from 0 $180^{\circ}$ were represented uniformly across Pyr neuron number $1-350$. The tuning curve of each neuron followed a Gaussian distribution with a standard deviation of $42^{\circ}$ (Bensmaia et al., 2008), and was implemented by varying the number of thalamic input synapses (at peak of gaussian: $\mathrm{N}_{\text {syn,Pyr }}=45$ synapses). A similar stimulation paradigm was applied also to the PV and VIP interneurons (peak $\mathrm{N}_{\text {syn,PV }}=30$ synapses, $\mathrm{N}_{\text {syn,VIP }}=30$ synapses), although with a broader tuning curve (standard deviation of $147^{\circ}$ and $294^{\circ}$, respectively; Wang et al., 2004) which allowed sufficient activation of PV and VIP interneurons for SST neuron suppression (Gentet et al., 2012; Muñoz et al., 2017). Across random seeds, we additionally applied a non-systematic noise of $\pm 30 \%$ 
(sampled from a uniform distribution) to the number of thalamic input synapses, corresponding to input errors e.g. due to finger position and in tactile surface irregularities, as well as noise in the signal progression along the CNS hierarchy from the periphery. We also tested a condition where synapses were placed on apical dendrites instead of basal dendrites, using 67 synapses to elicit an equivalent average response rate as in the regular basal input condition of the microcircuits with younger Pyr neurons. In addition, we tested a condition of low basal or apical input, using 31 and 45 synapses, respectively.

Classifier Models. Discrimination accuracy using the microcircuit model outputs to $85^{\circ}$ vs. $95^{\circ}$ stimulus orientations was assessed for microcircuits with younger vs older Pyr neuron models using classifier models available in the scikit-learn Python module. We used angles symmetrically close to $90^{\circ}$ because this angle corresponded to the middle-preference for the neuronal population. For each microcircuit model with younger and older Pyr neurons, stimulus discrimination was tested for a single circuit (i.e., fixed connectivity between the neurons) across 80 randomizations of background OU excitation and stimulus amplitude $\left(N_{s y n}\right)$. Output PSPs from each Pyr neuron during response ( 0 - $100 \mathrm{~ms}$ post-stimulus) were first computed by convolving the neuron output spike trains with an averaged AMPA $\left(\tau_{r}=0.5 \mathrm{~ms} ; \tau_{d}=3 \mathrm{~ms}\right)$ and NMDA $\left(\tau_{r}=2 \mathrm{~ms} ; \tau_{d}=65 \mathrm{~ms}\right)$ PSP waveform:

$$
P S P_{\frac{A M P A}{N M D A}}(t)=\frac{\left(e^{-t / \tau_{d, A M P A}}-e^{t / \tau_{r, A M P A}}\right)+\left(e^{-t / \tau_{d, N M D A}}-e^{t / \tau_{r, N M D A}}\right)}{2}
$$

We then computed the area under the curve of the resulting PSPs for each Pyr neuron and used it as feature input to the classifier models. We trained and tested with three different types of classifier models: 1) a linear support vector model, 2) a Gaussian naïve Bayes model, and 3) a multi-layer perceptron model (see Table S6 for full descriptions of classifier model input arguments). Training was done using a random subset of 15 of the 80 stimulus presentations per orientation and testing was done on the other 65 . We derived performance statistics for the classifiers using 300 random permutations of the stimulus presentations used for training and testing. We chose PSP area under curve as the feature inputs because this measure is representative of the amount of charge that downstream postsynaptic neurons will receive. 
PSP summation simulations. To investigate the effects of h-channel density changes with age on synaptic integration, we simulated PSP summation in the younger and older neuron models using a $50 \mathrm{~Hz}$ train of 5 step current pulses ( $2 \mathrm{~ms}, 3 \mathrm{nA}$ each) injected at a presynaptic neuron soma (Pyr, SST, or PV) to trigger a train of action potentials and consequently PSPs in a Pyr neuron. Connectivity parameters to the postsynaptic Pyr neuron were the same as in circuit simulations (Table S5), except for Pyr $\rightarrow$ Pyr neuron conductance, which was reduced to $0.3 \mathrm{nS}$ to prevent the postsynaptic Pyr neuron from spiking. We simulated 20 trials (with randomized synaptic probability) across 20 connections (randomized synaptic locations) and calculated the mean and 95\% confidence intervals across connections by bootstrapping (500 iterations) the trial means. We ran these simulations with and without DC current compensating for resting membrane potential differences between the younger and older neuron models. We compensated the resting membrane potential by injecting positive current in the younger Pyr neuron (6.65 pA) and negative current in the older Pyr neuron (-6.65 pA).

Human HCN1 channel transcriptomics data. We accessed transcriptomic data from the MTG of 8 human postmortem brains (Hodge et al., 2019; https://portal.brain-map.org/atlases-anddata/rnaseq/human-mtg-smart-seq; younger: $n=5$ donors, ages $24-48$ years; older: $n=3$ donors, ages 50 - 66 years). Data frames were prefiltered in Python to only include gene counts from glutamatergic neurons located in L5 and to combine intron and exon gene count data frames. We analyzed gene expression using the Seurat v3 toolkit in R (Stuart et al., 2019), where we lognormalized gene counts by dividing each count by the total count for that cell, scaling this by a factor of 10,000, and then computing the natural log-transform using the log1p function. Lognormalized counts for HCN1 transcripts were compared between younger and older cells using a Wilcoxon Ranked Sum test, and the log-fold change of average expression was computed.

Model Population Analysis. We analyzed the population parameter sets of younger and older Pyr neuron models generated from our multi-objective optimizations by first selecting sets of acceptable models based on performance (within 2 SD from the target experimental means, except 3 SD for AP width, AHP depth features, and AHP slow time). To narrow down our selection to models that captured sag voltage amplitude well, we set the SD threshold for this feature to $1 \mathrm{SD}$. After selecting the sets of models (younger =1,800 models; older =1,611 models), we normalized 
their conductance parameter values by the upper and lower search limits used in the optimization ( 0 corresponding to the lower limit and 1 corresponding to the upper limit).

\section{Acknowledgements}

AGM, SJT and EH thank the Krembil Foundation for their generous funding support. MW and EH thank NSERC for funding support. SJT and TAV also thank the generous support from CAMH Discovery Fund and Kavli Foundation. We also thank Dr. Yuxiao Chen for technical assistance in gene-expression analysis. As well, we are immensely grateful to our neurosurgical patients and their families for consenting to the use of their tissue samples for research. 


\section{References}

Albertson, A. J., Bohannon, A. S., \& Hablitz, J. J. (2017). HCN Channel Modulation of Synaptic Integration in GABAergic Interneurons in Malformed Rat Neocortex. Frontiers in Cellular Neuroscience, 11. https://doi.org/10.3389/fncel.2017.00109

Barthó, P., Hirase, H., Monconduit, L., Zugaro, M., Harris, K. D., \& Buzsáki, G. (2004). Characterization of Neocortical Principal Cells and Interneurons by Network Interactions and Extracellular Features. Journal of Neurophysiology, 92(1), 600-608. https://doi.org/10.1152/jn.01170.2003

Beaulieu-Laroche, L., Toloza, E. H. S., Goes, M.-S. van der, Lafourcade, M., Barnagian, D., Williams, Z. M., Eskandar, E. N., Frosch, M. P., Cash, S. S., \& Harnett, M. T. (2018). Enhanced Dendritic Compartmentalization in Human Cortical Neurons. Cell, 175(3), 643651.e14. https://doi.org/10.1016/j.cell.2018.08.045

Bensmaia, S. J., Denchev, P. V., Dammann, J. F., Craig, J. C., \& Hsiao, S. S. (2008). The Representation of Stimulus Orientation in the Early Stages of Somatosensory Processing. Journal of Neuroscience, 28(3), 776-786. https://doi.org/10.1523/JNEUROSCI.416207.2008

Bryson, A., Hatch, R. J., Zandt, B.-J., Rossert, C., Berkovic, S. F., Reid, C. A., Grayden, D. B., Hill, S. L., \& Petrou, S. (2020). GABA-mediated tonic inhibition differentially modulates gain in functional subtypes of cortical interneurons. Proceedings of the National Academy of Sciences, 117(6), 3192-3202. https://doi.org/10.1073/pnas.1906369117

Bucur, B., \& Madden, D. J. (2010). Effects of Adult Age and Blood Pressure on Executive Function and Speed of Processing. Experimental Aging Research, 36(2), 153-168. https://doi.org/10.1080/03610731003613482

Carnevale, N. T., \& Hines, M. L. (2006). The NEURON Book (1 edition). Cambridge University Press.

Chen, X., Shu, S., Schwartz, L. C., Sun, C., Kapur, J., \& Bayliss, D. A. (2010). Homeostatic Regulation of Synaptic Excitability: Tonic GABAA Receptor Currents Replace I h in Cortical Pyramidal Neurons of HCN1 Knock-Out Mice. Journal of Neuroscience, 30(7), 2611-2622. https://doi.org/10.1523/JNEUROSCI.3771-09.2010

Choi, J., Ku, B., You, Y. G., Jo, M., Kwon, M., Choi, Y., Jung, S., Ryu, S., Park, E., Go, H., Kim, G., Cha, W., \& Kim, J. U. (2019). Resting-state prefrontal EEG biomarkers in correlation with MMSE scores in elderly individuals. Scientific Reports, 9(1), 10468. https://doi.org/10.1038/s41598-019-46789-2

Davis, H. P., Cohen, A., Gandy, M., Colombo, P., VanDusseldorp, G., Simolke, N., \& Romano, J. (1990). Lexical priming deficits as a function of age. Behavioral Neuroscience, 104(2), 288-297.

Destexhe, A., Rudolph, M., Fellous, J.-M., \& Sejnowski, T. J. (2001). Fluctuating synaptic conductances recreate in vivo-like activity in neocortical neurons. Neuroscience, 107(1), 13-24. https://doi.org/10.1016/S0306-4522(01)00344-X

Donoghue, T., Haller, M., Peterson, E. J., Varma, P., Sebastian, P., Gao, R., Noto, T., Lara, A. H., Wallis, J. D., Knight, R. T., Shestyuk, A., \& Voytek, B. (2020). Parameterizing neural power spectra into periodic and aperiodic components. Nature Neuroscience, 23(12), 16551665. https://doi.org/10.1038/s41593-020-00744-x 
Florez, C. M., McGinn, R. J., Lukankin, V., Marwa, I., Sugumar, S., Dian, J., Hazrati, L.-N., Carlen, P. L., Zhang, L., \& Valiante, T. A. (2015). In Vitro Recordings of Human Neocortical Oscillations. Cerebral Cortex, 25(3), 578-597. https://doi.org/10.1093/cercor/bht235

Fuhrmann, G., Segev, I., Markram, H., \& Tsodyks, M. (2002). Coding of Temporal Information by Activity-Dependent Synapses. Journal of Neurophysiology, 87(1), 140-148. https://doi.org/10.1152/jn.00258.2001

Gentet, L. J., Kremer, Y., Taniguchi, H., Huang, Z. J., Staiger, J. F., \& Petersen, C. C. H. (2012). Unique functional properties of somatostatin-expressing GABAergic neurons in mouse barrel cortex. Nature Neuroscience, 15(4), 607-612. https://doi.org/10.1038/nn.3051

Gidon, A., Zolnik, T. A., Fidzinski, P., Bolduan, F., Papoutsi, A., Poirazi, P., Holtkamp, M., Vida, I., \& Larkum, M. E. (2020). Dendritic action potentials and computation in human layer $2 / 3$ cortical neurons. Science, 367(6473), 83-87. https://doi.org/10.1126/science.aax6239

Goriounova, N. A., Heyer, D. B., Wilbers, R., Verhoog, M. B., Giugliano, M., Verbist, C., Obermayer, J., Kerkhofs, A., Smeding, H., Verberne, M., Idema, S., Baayen, J. C., Pieneman, A. W., de Kock, C. P., Klein, M., \& Mansvelder, H. D. (2018). Large and fast human pyramidal neurons associate with intelligence. ELife, 7, e41714. https://doi.org/10.7554/eLife.41714

Gouwens, N. W., Berg, J., Feng, D., Sorensen, S. A., Zeng, H., Hawrylycz, M. J., Koch, C., \& Arkhipov, A. (2018). Systematic generation of biophysically detailed models for diverse cortical neuron types. Nature Communications, 9(1), 710. https://doi.org/10.1038/s41467-017-02718-3

Hagen, E., Næss, S., Ness, T. V., \& Einevoll, G. T. (2018). Multimodal Modeling of Neural Network Activity: Computing LFP, ECoG, EEG, and MEG Signals With LFPy 2.0. Frontiers in Neuroinformatics, 12. https://doi.org/10.3389/fninf.2018.00092

Halgren, M., Fabó, D., Ulbert, I., Madsen, J. R., Erőss, L., Doyle, W. K., Devinsky, O., Schomer, D., Cash, S. S., \& Halgren, E. (2018). Superficial Slow Rhythms Integrate Cortical Processing in Humans. Scientific Reports, 8(1), 2055. https://doi.org/10.1038/s41598-018-20662-0

Hay, E., Hill, S., Schürmann, F., Markram, H., \& Segev, I. (2011). Models of Neocortical Layer 5b Pyramidal Cells Capturing a Wide Range of Dendritic and Perisomatic Active Properties. PLoS Comput Biol, 7(7), e1002107. https://doi.org/10.1371/journal.pcbi.1002107

Hay, E., Schürmann, F., Markram, H., \& Segev, I. (2013). Preserving axosomatic spiking features despite diverse dendritic morphology. Journal of Neurophysiology, 109(12), 2972-2981. https://doi.org/10.1152/jn.00048.2013

Hay, E., \& Segev, I. (2015). Dendritic Excitability and Gain Control in Recurrent Cortical Microcircuits. Cerebral Cortex, 25(10), 3561-3571. https://doi.org/10.1093/cercor/bhu200

Hodge, R. D., Bakken, T. E., Miller, J. A., Smith, K. A., Barkan, E. R., Graybuck, L. T., Close, J. L., Long, B., Johansen, N., Penn, O., Yao, Z., Eggermont, J., Höllt, T., Levi, B. P., Shehata, S. I., Aevermann, B., Beller, A., Bertagnolli, D., Brouner, K., ... Lein, E. S. (2019). Conserved cell types with divergent features in human versus mouse cortex. Nature, 1-8. https://doi.org/10.1038/s41586-019-1506-7

Kalmbach, B. E., Buchin, A., Long, B., Close, J., Nandi, A., Miller, J. A., Bakken, T. E., Hodge, R. D., Chong, P., de Frates, R., Dai, K., Maltzer, Z., Nicovich, P. R., Keene, C. D., Silbergeld, D. L., Gwinn, R. P., Cobbs, C., Ko, A. L., Ojemann, J. G., ... Ting, J. T. (2018). H-Channels 
Contribute to Divergent Intrinsic Membrane Properties of Supragranular Pyramidal Neurons in Human versus Mouse Cerebral Cortex. Neuron, 100(5), 1194-1208.e5. https://doi.org/10.1016/j.neuron.2018.10.012

Kalmbach, B. E., Hodge, R. D., Jorstad, N. L., Owen, S., Frates, R. de, Yanny, A. M., Dalley, R., Mallory, M., Graybuck, L. T., Radaelli, C., Keene, C. D., Gwinn, R. P., Silbergeld, D. L., Cobbs, C., Ojemann, J. G., Ko, A. L., Patel, A. P., Ellenbogen, R. G., Bakken, T. E., ... Ting, J. T. (2021). Signature morpho-electric, transcriptomic, and dendritic properties of human layer 5 neocortical pyramidal neurons. Neuron, 109(18), 2914-2927.e5. https://doi.org/10.1016/j.neuron.2021.08.030

Kole, M. H. P., Hallermann, S., \& Stuart, G. J. (2006). Single Ih Channels in Pyramidal Neuron Dendrites: Properties, Distribution, and Impact on Action Potential Output. Journal of Neuroscience, 26(6), 1677-1687. https://doi.org/10.1523/JNEUROSCI.3664-05.2006

Legge, G. E., Granquist, C., Lubet, A., Gage, R., \& Xiong, Y.-Z. (2019). Preserved tactile acuity in older pianists. Attention, Perception, \& Psychophysics, 81(8), 2619-2625. https://doi.org/10.3758/s13414-019-01844-y

Legge, G. E., Madison, C., Vaughn, B. N., Cheong, A. M. Y., \& Miller, J. C. (2008). Retention of high tactile acuity throughout the life span in blindness. Perception \& Psychophysics, 70(8), 1471-1488. https://doi.org/10.3758/PP.70.8.1471

Mäki-Marttunen, T., Krull, F., Bettella, F., Hagen, E., Næss, S., Ness, T. V., Moberget, T., Elvsåshagen, T., Metzner, C., Devor, A., Edwards, A. G., Fyhn, M., Djurovic, S., Dale, A. M., Andreassen, O. A., \& Einevoll, G. T. (2019). Alterations in Schizophrenia-Associated Genes Can Lead to Increased Power in Delta Oscillations. Cerebral Cortex, 29(2), 875891. https://doi.org/10.1093/cercor/bhy291

McIntosh, A. R., Vakorin, V., Kovacevic, N., Wang, H., Diaconescu, A., \& Protzner, A. B. (2014). Spatiotemporal Dependency of Age-Related Changes in Brain Signal Variability. Cerebral Cortex, 24(7), 1806-1817. https://doi.org/10.1093/cercor/bht030

Mohan, A., Thalamuthu, A., Mather, K. A., Zhang, Y., Catts, V. S., Weickert, C. S., \& Sachdev, P. S. (2018). Differential expression of synaptic and interneuron genes in the aging human prefrontal cortex. Neurobiology of Aging, 70, 194-202. https://doi.org/10.1016/j.neurobiolaging.2018.06.011

Mohan, H., Verhoog, M. B., Doreswamy, K. K., Eyal, G., Aardse, R., Lodder, B. N., Goriounova, N. A., Asamoah, B., B. Brakspear, A. B. C., Groot, C., van der Sluis, S., Testa-Silva, G., Obermayer, J., Boudewijns, Z. S. R. M., Narayanan, R. T., Baayen, J. C., Segev, I., Mansvelder, H. D., \& de Kock, C. P. J. (2015). Dendritic and Axonal Architecture of Individual Pyramidal Neurons across Layers of Adult Human Neocortex. Cerebral Cortex, 25(12), 4839-4853. https://doi.org/10.1093/cercor/bhv188

Moradi Chameh, H., Rich, S., Wang, L., Chen, F.-D., Zhang, L., Carlen, P. L., Tripathy, S. J., \& Valiante, T. A. (2021). Diversity amongst human cortical pyramidal neurons revealed via their sag currents and frequency preferences. Nature Communications, 12(1), 2497. https://doi.org/10.1038/s41467-021-22741-9

Muñoz, W., Tremblay, R., Levenstein, D., \& Rudy, B. (2017). Layer-specific modulation of neocortical dendritic inhibition during active wakefulness. Science, 355(6328), 954-959. https://doi.org/10.1126/science.aag2599 
Pegasiou, C. M., Zolnourian, A., Gomez-Nicola, D., Deinhardt, K., Nicoll, J. A. R., Ahmed, A. I., Vajramani, G., Grundy, P., Verhoog, M. B., Mansvelder, H. D., Perry, V. H., Bulters, D., \& Vargas-Caballero, M. (2020). Age-Dependent Changes in Synaptic NMDA Receptor Composition in Adult Human Cortical Neurons. Cerebral Cortex, 30(7), 4246-4256. https://doi.org/10.1093/cercor/bhaa052

Peters, R. (2006). Ageing and the brain. Postgraduate Medical Journal, 82(964), 84-88. https://doi.org/10.1136/pgmj.2005.036665

Prevot, T. D., Sumitomo, A., Tomoda, T., Knutson, D. E., Li, G., Mondal, P., Banasr, M., Cook, J. M., \& Sibille, E. (2021). Reversal of Age-Related Neuronal Atrophy by $\alpha 5-G A B A A$ Receptor Positive Allosteric Modulation. Cerebral Cortex, 31(2), 1395-1408. https://doi.org/10.1093/cercor/bhaa310

Prönneke, A., Scheuer, B., Wagener, R. J., Möck, M., Witte, M., \& Staiger, J. F. (2015). Characterizing VIP Neurons in the Barrel Cortex of VIPcre/tdTomato Mice Reveals LayerSpecific Differences. Cerebral Cortex, 25(12), 4854-4868. https://doi.org/10.1093/cercor/bhv202

Ramaswamy, S., Courcol, J.-D., Abdellah, M., Adaszewski, S. R., Antille, N., Arsever, S., Atenekeng, G., Bilgili, A., Brukau, Y., Chalimourda, A., Chindemi, G., Delalondre, F., Dumusc, R., Eilemann, S., Gevaert, M. E., Gleeson, P., Graham, J. W., Hernando, J. B., Kanari, L., ... Markram, H. (2015). The neocortical microcircuit collaboration portal: A resource for rat somatosensory cortex. Frontiers in Neural Circuits, 44. https://doi.org/10.3389/fncir.2015.00044

Ramos, B. P., Stark, D., Verduzco, L., Dyck, C. H. van, \& Arnsten, A. F. T. (2006). A2Aadrenoceptor stimulation improves prefrontal cortical regulation of behavior through inhibition of cAMP signaling in aging animals. Learning \& Memory, 13(6), 770-776. https://doi.org/10.1101/Im.298006

Rich, S., Moradi Chameh, H., Sekulic, V., Valiante, T. A., \& Skinner, F. K. (2021). Modeling Reveals Human-Rodent Differences in H-Current Kinetics Influencing Resonance in Cortical Layer 5 Neurons. Cerebral Cortex, 31(2), 845-872. https://doi.org/10.1093/cercor/bhaa261

Roopun, A. K., Middleton, S. J., Cunningham, M. O., LeBeau, F. E. N., Bibbig, A., Whittington, M. A., \& Traub, R. D. (2006). A beta2-frequency $(20-30 \mathrm{~Hz})$ oscillation in nonsynaptic networks of somatosensory cortex. Proceedings of the National Academy of Sciences, 103(42), 15646-15650. https://doi.org/10.1073/pnas.0607443103

Roth, F. C., \& Hu, H. (2020). An axon-specific expression of HCN channels catalyzes fast action potential signaling in GABAergic interneurons. Nature Communications, 11(1), 2248. https://doi.org/10.1038/s41467-020-15791-y

Scimemi, A., Andersson, A., Heeroma, J. H., Strandberg, J., Rydenhag, B., McEvoy, A. W., Thom, M., Asztely, F., \& Walker, M. C. (2006). Tonic GABAA receptor-mediated currents in human brain. European Journal of Neuroscience, 24(4), 1157-1160. https://doi.org/10.1111/j.1460-9568.2006.04989.x

Seeman, S. C., Campagnola, L., Davoudian, P. A., Hoggarth, A., Hage, T. A., Bosma-Moody, A., Baker, C. A., Lee, J. H., Mihalas, S., Teeter, C., Ko, A. L., Ojemann, J. G., Gwinn, R. P., Silbergeld, D. L., Cobbs, C., Phillips, J., Lein, E., Murphy, G., Koch, C., ... Jarsky, T. (2018). 
Sparse recurrent excitatory connectivity in the microcircuit of the adult mouse and human cortex. ELife, 7, e37349. https://doi.org/10.7554/eLife.37349

Sermet, B. S., Truschow, P., Feyerabend, M., Mayrhofer, J. M., Oram, T. B., Yizhar, O., Staiger, J. F., \& Petersen, C. C. (2019). Pathway-, layer- and cell-type-specific thalamic input to mouse barrel cortex. ELife, 8, e52665. https://doi.org/10.7554/eLife.52665

Sivagnanam, S., Majumdar, A., Yoshimoto, K., Astakhov, V., B, A., Martone, M., \& Carnevale, N. T. (2013). Introducing The Neuroscience Gateway: Vol. 993 of CEUR Workshop Proceedings. http://ceur-ws.org/Vol-993/paper10.pdf

Song, C., \& Moyer, J. R. (2017). Layer- and subregion-specific differences in the neurophysiological properties of rat medial prefrontal cortex pyramidal neurons. Journal of Neurophysiology, 119(1), 177-191. https://doi.org/10.1152/jn.00146.2017

Song, W., \& Semework, M. (2015). Tactile representation in somatosensory thalamus (VPL) and cortex (S1) of awake primate and the plasticity induced by VPL neuroprosthetic stimulation. Brain Research, 1625, 301-313. https://doi.org/10.1016/j.brainres.2015.08.046

Stuart, T., Butler, A., Hoffman, P., Hafemeister, C., Papalexi, E., Mauck, W. M., Hao, Y., Stoeckius, M., Smibert, P., \& Satija, R. (2019). Comprehensive Integration of Single-Cell Data. Cell, 177(7), 1888-1902.e21. https://doi.org/10.1016/j.cell.2019.05.031

Thakur, P. H., Fitzgerald, P. J., Lane, J. W., \& Hsiao, S. S. (2006). Receptive Field Properties of the Macaque Second Somatosensory Cortex: Nonlinear Mechanisms Underlying the Representation of Orientation Within a Finger Pad. Journal of Neuroscience, 26(52), 13567-13575. https://doi.org/10.1523/JNEUROSCI.3990-06.2006

Van Geit, W., Gevaert, M., Chindemi, G., Rössert, C., Courcol, J.-D., Muller, E. B., Schürmann, F., Segev, I., \& Markram, H. (2016). BluePyOpt: Leveraging Open Source Software and Cloud Infrastructure to Optimise Model Parameters in Neuroscience. Frontiers in Neuroinformatics, 10. https://doi.org/10.3389/fninf.2016.00017

Wang, M., Gamo, N. J., Yang, Y., Jin, L. E., Wang, X.-J., Laubach, M., Mazer, J. A., Lee, D., \& Arnsten, A. F. T. (2011). Neuronal basis of age-related working memory decline. Nature, 476(7359), 210-213. https://doi.org/10.1038/nature10243

Wang, M., Ramos, B. P., Paspalas, C. D., Shu, Y., Simen, A., Duque, A., Vijayraghavan, S., Brennan, A., Dudley, A., Nou, E., Mazer, J. A., McCormick, D. A., \& Arnsten, A. F. T. (2007). A2A-Adrenoceptors Strengthen Working Memory Networks by Inhibiting cAMPHCN Channel Signaling in Prefrontal Cortex. Cell, 129(2), 397-410. https://doi.org/10.1016/j.cell.2007.03.015

Wang, X.-J., Tegnér, J., Constantinidis, C., \& Goldman-Rakic, P. S. (2004). Division of labor among distinct subtypes of inhibitory neurons in a cortical microcircuit of working memory. Proceedings of the National Academy of Sciences, 101(5), 1368-1373. https://doi.org/10.1073/pnas.0305337101

Williams, S. R., \& Stuart, G. J. (2000). Site Independence of EPSP Time Course Is Mediated by Dendriticl h in Neocortical Pyramidal Neurons. Journal of Neurophysiology, 83(5), 31773182. https://doi.org/10.1152/jn.2000.83.5.3177

Yao, H. K., Guet-McCreight, A., Mazza, F., Chameh, H. M., Prevot, T. D., Griffiths, J. D., Tripathy, S. J., Valiante, T. A., Sibille, E., \& Hay, E. (2022). Reduced inhibition in depression impairs 
stimulus processing in human cortical microcircuits. Cell Reports, 38(2).

https://doi.org/10.1016/j.celrep.2021.110232

$\mathrm{Yu}, \mathrm{J.,}$ Hu, H., Agmon, A., \& Svoboda, K. (2019). Recruitment of GABAergic Interneurons in the Barrel Cortex during Active Tactile Behavior. Neuron, 104(2), 412-427.e4. https://doi.org/10.1016/j.neuron.2019.07.027

Zurita, H., Feyen, P. L. C., \& Apicella, A. J. (2018). Layer 5 Callosal Parvalbumin-Expressing Neurons: A Distinct Functional Group of GABAergic Neurons. Frontiers in Cellular Neuroscience, 12. https://doi.org/10.3389/fncel.2018.00053 